\title{
Absence of the discontinuous transition in the one-dimensional triplet creation model
}

\author{
Su-Chan Park \\ Institut für Theoretische Physik, Universität zu Köln, Zülpicher Str. 77, 50937 Köln, Germany
}

(Dated: November 19, 2018)

\begin{abstract}
Although Hinrichsen in his unpublished work theoretically rebutted the possibility of the discontinuous transition in one-dimensional nonequilibrium systems unless there are additional conservation laws, long-range interactions, macroscopic currents, or special boundary conditions, we have recently observed the resurrection of the claim that the triplet creation model (TC) introduced by Dickman and Tomé [Phys. Rev. E 44, 4833 (1991)] would show the discontinuous transition. By extensive simulations, however, we find that the one-dimensional TC does belong to the directed percolation universality class even for larger diffusion constant than the suggested tricritical point in the literature. Furthermore, we find that the phase boundary is well described by the crossover from the mean field to the directed percolation, which supports the claim that the one-dimensional TC does not exhibit a discontinuous transition.
\end{abstract}

PACS numbers: 05.70.Ln,64.60.De,05.70.Fh

\section{INTRODUCTION}

Although the field theory for the tricritical phenomena in reaction-diffusion systems was developed more than two decades ago [1, 2] (see also Ref. [3]), not many numerical studies have followed [4,, 5$]$. One apparent reason is the numerical difficulty, but it can be soon overcome by the increasing computing power. More seriously, it was strongly argued that no discontinuous transition is possible in one dimension once there are no additional conservation laws, long-range interactions, macroscopic currents, or special boundary conditions [ 6$]$, which rebutted the observed discontinuous transition in one-dimensional triplet creation model (TC) by Dickman and Tomé [7]. In view of the fact that large portion of the studies on the absorbing phase transitions (for a review, see, e.g. Refs. [8, 9, 10]) is focused on the systems in one dimension, this theory presumably has kept researchers from being into the tricritical phenomena.

Recently, however, numerical studies in favor of the original claim by Dickman and Tomé have been reported [11, 12, 13]. If this claim rather than the theory in Ref. [6] turns out to be right, we would observe an avalanche of studies on the tricritical phenomena. Unfortunately, however, no theoretical argument regarding the mechanism to stabilize a domain in one dimension has been suggested as yet. Moreover, the tricritical point of the diffusion rate reported in Ref. [11, 12] is too large to reject the opinion that the system will eventually crossover to the directed percolation (DP) universality class after a long transient time. Recent study by Ferreira and Fontanari [14] using $n$-site approximation alluded to the crossover rather than the tricritical behavior, though they did not strongly put forward such a scenario because of the computational limitation of their method. Actually, Hinrichsen [6] numerically showed that the simulation time in Ref. 7] was too short to see the correct scaling behavior. Interestingly, Cardozo and Fontanari [12] also refuted the value of the tricritical point originally suggested. Hence, if the argument by Hinrichsen [6] is right, it is very probable that more extensive simulations than those in Ref. [12] would revive the history.

Indeed, we found the DP scaling over the parameter range where the tricritical point was located by Cardozo and Fontanari [12]. This paper is for providing numerical evidences to support the theory suggested by Hinrichsen [6].

The rest of this paper is organized as follows: Section IIintroduces the $d$-dimensional TC and explains the algorithm implemented for numerical simulations. The numerical results showing the DP scaling behavior for larger diffusion rate than the previously reported tricritical point will be presented in Sec. III] In Sec. IV] we will argue that there is a crossover rather than a tricriticality in one dimension by studying the behavior of the phase boundary. Section $\nabla$ summarizes the work.

\section{TRIPLET CREATION MODEL}

The TC is an interacting hard core particles system on a $d$-dimensional hypercubic lattice with three processes, hopping (with rate $D$ ), spontaneous annihilation (with rate $\gamma$ ), and creation by a triplet (with rates $s$ ) 7]. By hard core is meant that no two particles can occupy the same site at the same time. By a suitable time rescaling, we can set $D+\gamma+s=1$ without loss of generality. It is also convenient to introduce the annihilation probability $p$ such that $\gamma=(1-D) p$ and $s=(1-D)(1-p)$. The detailed dynamics is to be explained in terms of the algorithm used for simulations.

At time $t, N_{t}$ particles are distributed on a $d$ dimensional hypercube of size $L^{d}\left(N_{t}\right.$ is a random variable). Each site is represented by a lattice vector $\boldsymbol{m}=$ $\left(m_{1}, \ldots, m_{d}\right)\left(0 \leq m_{i} \leq L-1\right)$. The unit vector along direction $i$ is denoted by $\boldsymbol{e}_{i}(i=1, \ldots, d)$. In all simulations in this paper, a fully occupied initial condition and periodic boundary conditions are assumed.

The algorithm begins with a random selection of a particle in the system. For convenience, let us refer to the 
lattice vector of the site the selected particle resides as $\boldsymbol{m}$. With probability $D$, hopping is attempted to a target site which is chosen randomly among $2 d$ nearest neighbors of the site $\boldsymbol{m}$. This hopping is successful only if the target site is empty (hard core exclusion), otherwise there is no configuration change. With probability $1-D$, either annihilation (with probability $p$ ) or creation (with probability $1-p$ ) will be attempted. When annihilation is decided, the selected particle will be irreversibly removed from the system. If the creation is to occur, one of the directions $i(i=1, \ldots, d)$ is selected randomly. Two sites $\boldsymbol{m}+\boldsymbol{e}_{i}$ and $\boldsymbol{m}+2 \boldsymbol{e}_{i}$ are checked whether both sites are occupied or not. If both sites are also occupied, one of the two sites, $\boldsymbol{m}+3 \boldsymbol{e}_{i}$ or $\boldsymbol{m}-\boldsymbol{e}_{i}$, is chosen at random as a target site and a new particle is created at the target site provided it is empty. If any of the conditions for creation is not satisfied, nothing happens. After an attempt to change a configuration, time increases by $1 / N_{t}$ regardless of its success. The above procedure iterates until either the system reaches the absorbing state where no particle remains or time gets larger than the preassigned observation time.

When $d=1$, the above dynamics is exactly the same as that in Ref. 12] with $p=1 /(1+\lambda)$. This is because the probability to find three occupied sites in a row does not depend on whether two sites $\boldsymbol{m}+\boldsymbol{e}_{i}$ and $\boldsymbol{m}+2 \boldsymbol{e}_{i}$ (in this work) or $\boldsymbol{m}+\boldsymbol{e}_{i}$ and $\boldsymbol{m}-\boldsymbol{e}_{i}$ (in Ref. [12]) are examined.

Although we introduced $d$-dimensional TC, discussions from Sec. III on will be focused only on the onedimensional model. Thus, the dimensionality of the model will not be mentioned explicitly in the following.

In the simulation, we measure the particle density $\rho(t)=\left\langle N_{t}\right\rangle / L$, where $\langle\ldots\rangle$ means the average over independent realizations, and the (survival) probability that there is a particle in the system at time $t$. The measurement of the survival probability is mainly for making sure that the system is large enough not to be affected by the finite size effect up to the observation time.

\section{CRITICAL DENSITY DECAY}

This section studies the critical behavior of the TC with $D=0.95$ and $D=0.98$. Since the question in this section is whether the TC shows the discontinuous transition or the continuous transition governed by the DP fixed point, observing the DP scaling behavior of a single quantity is enough for our purpose. Anticipating the conclusion, we only study how the density decays near criticality.

Figure 1 depicts $\rho(t) t^{\delta}$ as a function of $t$ in semilogarithmic scales, where $\delta=0.1595$ is the critical density decay exponent of the DP class taken from Ref. [15]. The system size in the simulations for $D=0.95(0.98)$ is $L=2^{18}\left(2^{17}\right)$. The number of independent runs for each data set varies from $16(D=0.95$ and $p=0.08989)$ to $100(D=0.98$ and $p=0.094226)$. The system evolves

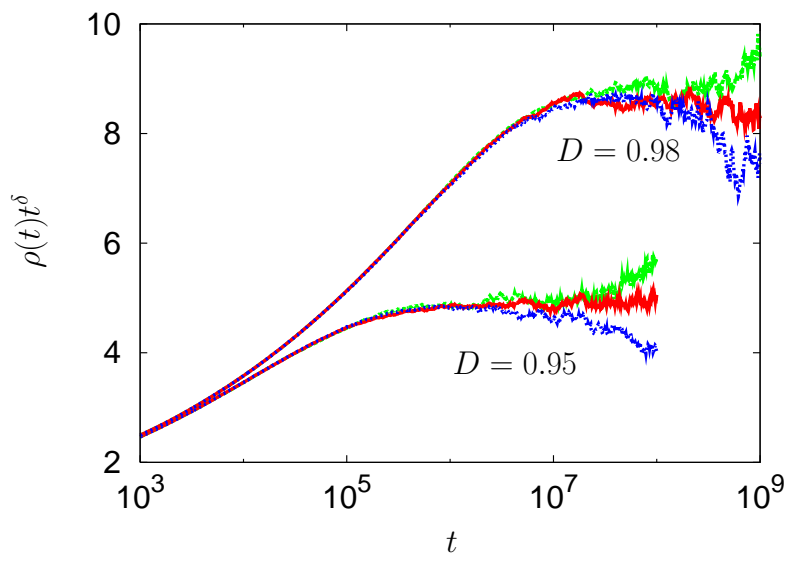

FIG. 1: (color online) Plots of $\rho(t) t^{\delta}$ vs $t$ with $\delta=0.1595$ (critical exponent of the DP class) for $D=0.95$ (lower three curves) and $D=0.98$ (upper three curves) in semi-logarithmic scales. The values of $p$ for $D=0.95$ are $0.08989,0.089895$, and 0.0899 from top to bottom. The values of $p$ for $D=0.98$ are $0.094224,0.094226$, and 0.094228 from top to bottom.

up to $t=10^{9}$ at the longest and no sample falls into the absorbing state during simulations. For $D=0.95$, the curve corresponding to $p=0.08989$ (0.0899) veers up (down), which indicates that the system is in the active (absorbing) phase. At $p=0.089895$, the curve is flat for more than two log-decades. Hence we conclude that the TC with $D=0.95$ belongs to the DP class with critical point $p_{c}=0.089895(5)$, where the number in parentheses indicates the error of the last digit. If we write the critical point using $\gamma=(1-D) p$, we get $\gamma_{c}=0.0044948(3)$ which should be compared with 0.004 50(1) reported in Ref. [12]. One should note that the DP scaling is observable from $t=10^{6}$ which is the end point of the simulation for $D=0.95$ in Ref. [12] (see Fig. 3(b) of Ref. [12]). Likewise, the simulation results for $D=0.98$ show the similar behavior as those for $D=0.95$ (see upper three curves in Fig. 11). The critical point for $D=0.98$ is found to be $p_{c}=0.094226(2)$ or $\gamma_{c}=0.001888452(4)$. In Ref. [12], the critical value $\gamma_{c}$ for $D=0.98$ was reported as $0.001886(2)$ and the simulation was terminated around $t=10^{7}$ from when the DP scaling is observable.

To conclude this section, the TC up to $D=0.98$ belongs to the DP class and previous claim of the existence of the tricritical point below $D=0.98$ is refuted. Our results also explain why Cardozo and Fontanari [12] observed continuously varying exponents as well as the compact growth; the system was analyzed before the correct scaling behavior was detected.

\section{CROSSOVER FROM THE MEAN FIELD TO THE DIRECTED PERCOLATION}

In Sec. III, we numerically confirmed that up to $D=$ 0.98 the TC does show continuous transition governed 
by the DP fixed point. Although this refuted the previous claim [11, 12] that the transition nature changes at a certain $D$ smaller than 0.98 , the possibility of the discontinuous transition at $0.98<D<1$ is still open. To provide an evidence that the DP fixed point governs the critical behavior beyond $D=0.98$, this section studies the phase boundary near $D=1$ with the focus on a possible crossover.

The investigation of the phase boundary to settle a controversy is not without precedent. The present author and his collaborator tried to resolve the controversy around the pair contact process with diffusion (for a review, see, e.g., Refs. [16, 17]) by studying the phase boundary of the crossover models [18]. Although this study could not elicit a full consensus, it certainly gives a hint about the system. So we think it is worth while to investigate the phase boundary of the TC. But, in Sec. III we have presented the simulation results only for $D=0.95$ and $D=0.98$, which is certainly not enough to see the structure of the phase boundary. For a better bird's-eye view, we will include some other critical points for $D<0.95$ even though we will not be presenting details.

Before directly analyzing the phase boundary, we will discuss some features of it which can be inferred without resorting to the nature of the transition. First, the transition point $p_{c}(D)$ is argued to be an increasing function of $D$. Second, $p_{c}(D)$ is expected to approach to the transition point of the mean field (MF) theory as $D \rightarrow 1$. By the MF theory in this paper is exclusively meant the onesite approximation (for a detail, one may consult Sec. 3 of Ref. [14])

$$
\frac{d \rho}{d \tau}=-p \rho+(1-p) \rho^{3}(1-\rho),
$$

which exhibits a discontinuous transition with the transition point $p_{0}=\frac{4}{31}$.

To argue that $p_{c}(D)$ increases with $D$, it is convenient to modify the TC by rescaling time $\tau \equiv(1-D) t$. Note that for $D<1$, however close $D$ is to 1 , the steady state property of the modified model is identical to the original one. In this modified model, a single particle dies out with rate $p$ and a triplet attempts to branch an offspring with rate $1-p$. Obviously, these rates are not dependent on $D$. The diffusion rate is now $\tilde{D} \equiv D /(1-D)$ which is an increasing function of $D$. Since the annihilation occurs regardless of the environment of a to-be-annihilated particle, the diffusion cannot directly affect the particle number fluctuation due to the annihilation. On the other hand, the branching is influenced by the diffusion, since a triplet can be either broken or newly formed by the movement of particles. Hence, whether the diffusion enhances or reduces the activity of the system for given $p$ can be answered by understanding if the diffusion will increase or decrease the number of active triplets, namely, triplets with a vacant neighbor.

Due to the hard core exclusion, a cluster of particles will lose one by the diffusion only at boundaries. How- ever, even if a cluster loses a single particle at a boundary, the number of active triplets does not decrease if the size of the cluster is larger than 3 (it can even increase the number of active triplets if the configuration change due to the diffusion is like $01101111 \rightarrow 01110111$, where 1 stands for a particle and 0 for a vacancy). Thus, the reduction of the number of active triplets by the diffusion occurs in very restricted situations. On the other hand, the diffusion can mediate the formation of (active) triplets in the bulk of region where none exists. To sum up, the diffusion tends to enhance the activity, which entails that the transition point should increase with $D$.

Next, we will discuss the limiting value of $p_{c}(D)$ as $D \rightarrow 1$, which will be denoted by $\tilde{p}$. To find $\tilde{p}$, we start from arguing that the TC under the $D \rightarrow 1$ limit is deeply related to the MF theory. This connection makes sense only when time is suitably rescaled as before. With this time scale, the diffusion rate $\tilde{D}$ grows indefinitely as $D \rightarrow 1$, but the annihilation and creation rates remain finite. From now on, we will call the limit $D \rightarrow 1$ the fast diffusion limit and the modified TC with rescaled time is always assumed when we are discussing the fast diffusion limit.

A finite system under the fast diffusion limit can be interpreted as follows: Right after any reaction (either annihilation or creation), the system arrives at the steady state of the diffusion process in no time and remains there until another reaction occurs. In the steady state of the diffusion-only system, all possible configurations for given number of particles have equal probability. Hence, the probability that a site is vacant and its three consecutive neighbors are occupied at time $\tau$ is $(L-n)(n)_{3} /(L)_{4}$, where $L$ is the system size and $n$ is the number of particles in the system at time $\tau$, and $(m)_{k} \equiv m(m-1) \ldots(m-k+1)$. Of course, the probability that a site is occupied at time $\tau$ is $n / L$. Since these probabilities do not depend on where the site is located, the probability distribution is fully specified by the number of particles and the master equation of the TC under the fast diffusion limit is reduced to

$$
\partial_{\tau} P_{n}=a_{n+1} P_{n+1}+c_{n-1} P_{n-1}-\left(a_{n}+c_{n}\right) P_{n},
$$

where $a_{n} \equiv p n, c_{n} \equiv(1-p)(L-n)(n)_{3} /(L-1)_{3}$, and $P_{n}$, though the argument of it is not written explicitly, is the probability that there are $n$ particles at time $\tau$. For convenience, we set $P_{L+1}=P_{-1}=0$.

The meaning of $a_{n}$ and $c_{n}$ in Eq. (2) can be interpreted as follows: $a_{n}$ means each particle (there are $n$ particles) dies with rate $p$ and $c_{n}$ means that each vacant site (there are $L-n$ vacant sites) becomes occupied with rate (1$p)(n)_{3} /(L-1)_{3}$, which is the dynamic rule of the TC on a fully connected graph. Hence, the master equation of the TC on a fully connected graph is exactly Eq. (2). If we take the thermodynamic limit $(L \rightarrow \infty)$ to Eq. (21), the law of large numbers has the density follow Eq. (11), that is, the MF theory. Now it is clear why we used $\tau$ in Eq. (1) as a time parameter. For convenience and because of an obvious reason, we will refer to the $\mathrm{TC}$ on 


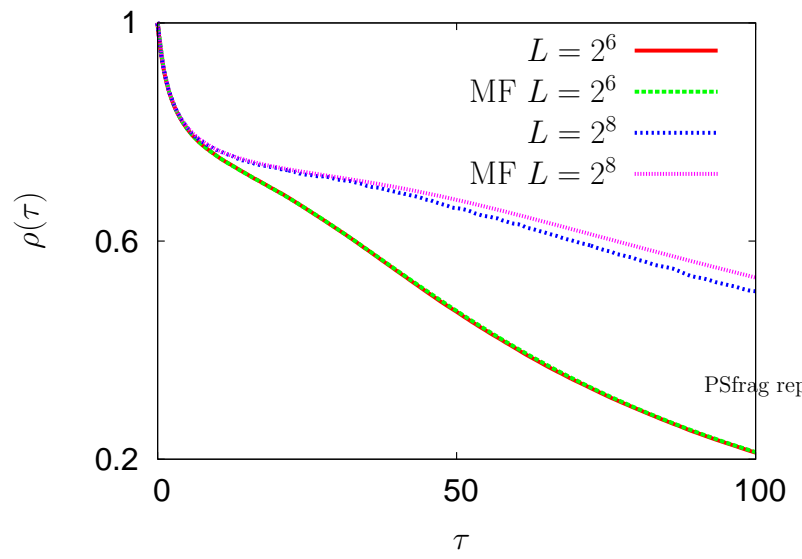

FIG. 2: (color online) Comparison of the numerical solutions of Eq. (2) with the simulation results of the TC with $1-$ $D=10^{-4}$. As explained in the text, the time is rescaled as $\tau=(1-D) t$. The systems sizes are $L=2^{6}$ (two below curves, though indiscernible) and $2^{8}$ (two above curves). $p$ is fixed at $\frac{1}{8}$. For $L=2^{6}$, no difference is detectable between simulation and the MF solution. On the other hand, the system with $L=2^{8}$ is distinct from the MF solution after $\tau=50$ (MF solution is slightly above the simulation results).

a fully connected graph with finite size as the MF model.

Although the fast diffusion limit was taken to arrive at the MF model in the above discussion, one can observe the behavior of the MF model even for nonzero $1-D$ once $(1-D) L^{2} \ll 1$ [7]. In Fig. 2, we compare the simulations of the TC for $1-D=10^{-4}$ to the (numerical) solutions of the MF model at $p=\frac{1}{8}$. The system sizes are $L=2^{6}$ and $L=2^{8}$. For the solutions of the MF model, we numerically integrate Eq. (2) with the initial condition $P_{n}(\tau=0)=\delta_{n L}(\delta$ here is the Kronecker delta symbol). The simulation results are not discernible from the behavior of the MF model for $L=2^{6}\left[(1-D) L^{2} \approx\right.$ 0.4], but clear deviation is observed when $L=2^{8}$.

In the above discussion, the thermodynamic limit, when necessary, is preceded by the fast diffusion limit. However, what we are interested in is the behavior of the $\mathrm{TC}$ with infinite size under the fast diffusion limit. That is, the thermodynamic limit should be taken before the fast diffusion limit. When two limits are involved in the calculation, one should be careful about which limit is taken first. Fortunately, the order of two limits are irrelevant in most cases. What is meant by most cases will become clear in due course.

Our discussion commences with the limiting behavior of the TC in the active phase $(p<\tilde{p})$. Let the critical diffusion probability for given $p$ be denoted by $D_{c}(p)$. Since $p_{c}(D)$ is a monotonous function, $D_{c}(p)$, if exists, is uniquely determined. If $1>D>D_{0}$, where $D_{0}$ is an arbitrary number strictly larger than $D_{c}(p)$, the system is in the active phase and the correlation length is bounded for all $D$ in this regime. If the system size is much larger than the bound of the correlation lengths in this regime, the behavior in the thermodynamic limit is observable for

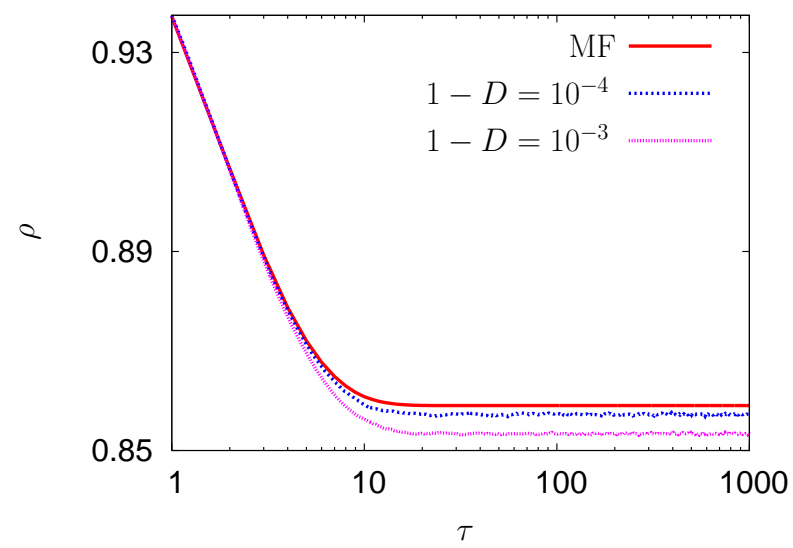

FIG. 3: (color online) Comparison of the numerical solutions of Eq. (1) with the simulation results of the TC with $1-D=$ $10^{-3}$ and $1-D=10^{-4}$ at $p=0.094226$. As in Fig. 2 $\tau$ is the rescaled time. The systems size for simulations is $L=2^{17}$. As $1-D$ decreases, the density approaches to the MF solution.

any $D_{0}<D<1$ even though the system is finite. Since we can always choose $D$ such that $(1-D) L^{2} \ll 1$ for given $L$, the TC under the fast diffusion limit preceded by the thermodynamic limit is identical to the MF theory at the same value of $p$.

To support this argument, Fig. 3 compares the MF theory with simulations of the TC for $D=0.999$ and $D=0.9999$ at $p=0.094226$ which is the critical point for $D=0.98$. Obviously, the density of the TC obtained from simulations approaches to the MF theory as $D \rightarrow 1$.

The above consideration reveals that $\tilde{p}$ should not be larger than $p_{0}$, otherwise the steady state density for $p_{0}<$ $p<\tilde{p}$ would decrease with $D$, which is contradictory to the role of the diffusion as a enhancer of the branching.

If $p>p_{0}$ where the TC as well as the MF model is in the absorbing phase, we can arrive at the same conclusion as above, that is, the behavior of the TC under the fast diffusion limit is not affected by whether before or after the thermodynamic limit is taken.

To complete our discussion, we first have to figure out if $\tilde{p}$ can be strictly smaller than $p_{0}$. Actually, this possibility was suggested by Fiore and de Oliveira [1] by extrapolating the phase boundary which was obtained numerically. Note that the discontinuity of a phase boundary per se is not an unrealistic conclusion. One can even find another report which, though in a different context, shows the discontinuity of the phase boundary [19].

However, this scenario does not seem plausible. To demonstrate why it is not likely, let us think about the situation where $\tilde{p}<p<p_{0},(1-D) L^{2} \ll 1$, and $L$ is much larger than the correlation length for given $p$ and $D$. As shown before, the system with $(1-D) L^{2} \ll 1$ is well described by the MF model. Since $L$ is assumed very large and this value of $p$ corresponds to the active phase of the MF theory, the MF model, and accordingly the TC itself with above mentioned parameters, stays at the steady state of the MF theory with nonzero density for long time 


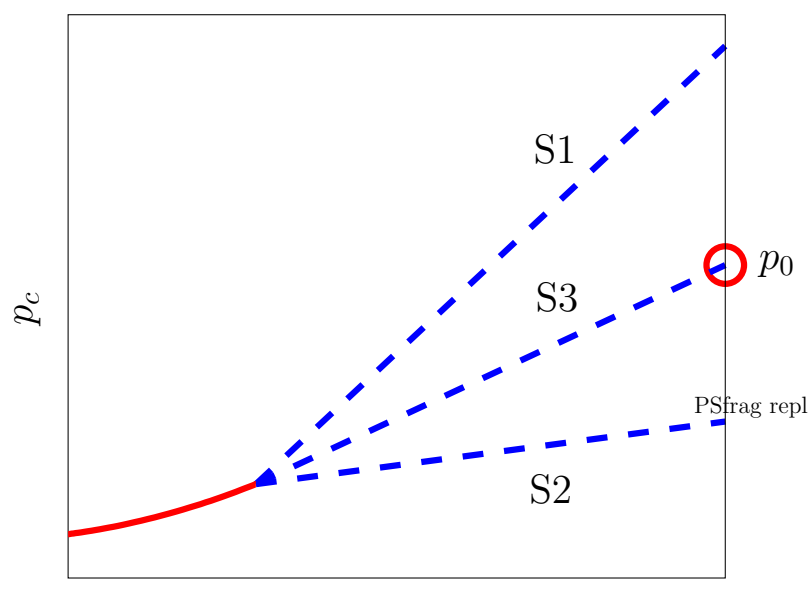

$D$

FIG. 4: (color online) Schematic representation of three scenarios regarding the limiting value of $p_{c}$ as $D \rightarrow 1 . p_{0}$ indicated by the open circle is the mean field transition point and scales in this figure are arbitrary. The broken lines depict the anticipated phase boundaries of each scenarios with known transition points depicted by the solid curve. The limiting value $\tilde{p}$ of each scenario is the point where the broken line meets the vertical line with $p_{0}$ on it. The first scenario (S1) is not acceptable because the steady state density should increase as $D$ increases (see the text). The second scenario (S2) which was proposed in Ref. [1] is possible only when there is a line of critical points (see the text), which does not seem plausible. Hence the last scenario (S3) is concluded to be the right one.

of the order of $O(\exp (L))$ (note that time in this context is the rescaled time $\tau$ ). On the other hand, the system size is assumed much larger than the correlation length and the system is in the absorbing phase by assumption, so the density should decay exponentially after time of the order of $O\left(L^{0}\right)$, which is contradictory to the previous consideration. Of course, if the correlation length in this regime diverges faster than $(1-D)^{-1 / 2}$ as $D \rightarrow 1$, the assumption $(1-D) L^{2} \ll 1$ with $L$ larger than the correlation length is not valid. However, this scenario of the diverging correlation length in this regime also does not seem plausible, because it suggests that the correlation length should diverge for $\tilde{p}<p<p_{0}$ and should become finite as soon as $p>p_{0}$. Hence, we conclude that $\tilde{p}$ is equal to $p_{0}$. The above discussion is summarized in Fig. 4

Up to now, we have not resorted to any assumptions about the transition nature. If we assume that the transition is always continuous, what can we say about the limiting behavior of the TC at $p=\tilde{p}=p_{0}$. In this case, the correlation length diverges as $(1-D)^{-\nu_{\perp}}$, where $\nu_{\perp} \simeq 1.09$ is the critical exponent of the DP class. Accordingly, it is not possible to think about the system size which is much larger than the correlation length but $(1-D) L^{2} \ll 1$ when $D$ is very close to 1 . That is, the fast diffusion and thermodynamic limit do not commute

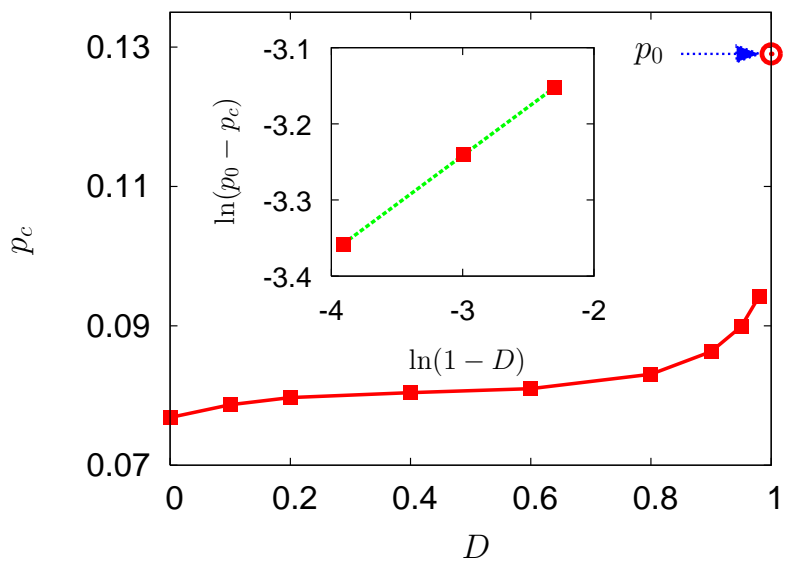

FIG. 5: (color online) Plot of $p_{c}$ as a function of $D$ for the TC. The MF transition point $p_{0}$ is indicated by an arrow. Inset: Plots of $\ln \left(p_{0}-p_{c}\right)$ vs $\ln (1-D)$ and its fitting function (see text). Symbols are from the simulations and the straight line is from the fitting.

at $p=p_{0}$. However, even if we assume that there is a discontinuous transition for finite $D$, the noncommutability of two limits at $p=p_{0}$ is still applicable, because the steady state density for $p=p_{0}$ is zero for any value of $D<1$, though the MF theory has a finite density at $p=p_{0}$. In a sense, this noncommutability is originated from the fact that the MF theory exhibits the discontinuous transition which is characterized by the discontinuity of the density at stationarity. At any rate, the thermodynamic limit commutes with the fast diffusion limit in most cases except at $p=p_{0}$.

Although the above consideration reveals that the phase boundary should approach to the MF transition point, it does not give any information about the transition nature. To get a nontrivial conclusion, the numerically obtained phase boundary will be examined.

Figure 5 depicts the phase boundary in $D-p$ plane. As argued before, the transition point increases with $D$. In the range $0 \leq D \leq 0.8$ where no controversy has ever been raised, the critical points do not change much, compared to the change from $D=0.8$ to $D=0.98$. It is likely that the phase boundary approaches to the MF transition point with infinite slope. If we fit the phase boundary using the fitting function

$$
\ln \left(p_{0}-p_{c}\right)=\frac{1}{\phi} \ln (1-D)+b,
$$

with two fitting parameters $\phi$ and $b$, we get $p_{c} \approx p_{0}-$ $0.057(1-D)^{0.127}(\phi \approx 8)$ from last three points [without $p_{0}$ and with $p_{c}=0.08630(1)$ for $D=0.9$; see Inset of Fig. 5 . Although the accuracy of the fitting should not be exaggerated, the clean power-law behavior shown in Inset of Fig. [5 strongly suggests that the phase boundary approaches to the MF transition point with infinite slope. Note that this infinite slope is the characteristics of the crossover behavior [20]. Also note that a "crossover" within a single universality class, which is actually not a 
crossover at all, does not show such a singular behavior [19]. Although we are considering the discontinuous transition, it is a natural generalization of the claim in Ref. 19] that no singularity can appear in the phase boundary along which only discontinuous transitions occur. Thus, the singular behavior of the phase boundary near $D=1$ supports that no discontinuous transition exists for $D<1$.

\section{SUMMARY AND DISCUSSION}

In summary, we investigated the triplet-creation model (TC) in one dimension. By extensive numerical simulations, we refuted the previous estimation of the tricritical point [11, 12]. We only observed the directed percolation scaling up to $D=0.98$ which is larger than the tricritical point suggested in Ref. [11, 12]. To go further beyond $D>0.98$, we analyzed the phase boundary near $D=1$. At first, we argued that the phase boundary should approach to the mean field transition point as $D \rightarrow 1$. Using this information, the phase boundary was analyzed using the power law fitting function Eq. (3) to find $\phi \simeq 8$, which, according to the general theory of the crossover [20], strongly suggests the absence of discontinuous transitions in one dimension.

As a final remark, we would like to comment on the conserved ensemble of the TC. In Ref. [11], Fiore and de Oliveira studied the conserved ensemble of the TC. They convincingly argued that the conserved version is equivalent to the TC studied in this paper, based on the proof in Refs. 21, 22]. This argument also embraces the limiting case $(D \rightarrow 1)$; see Sec. III in Ref. [1]. Actually, the equivalence becomes trivially true for the MF model under the thermodynamic limit because the equivalence criterion which is Eq. (30) of Ref. [22] becomes identical to the steady state condition of Eq. (11). Thus, the direct comparison of the results in this paper with those in Ref. [11] is fully legitimate although we studied the different ensemble. In this respect, this work clearly suggests a caveat. Unlike the belief regarding the merit of the conserved ensemble (for example, see Sec. V in Ref. 11]), one should study the conserved version with caution even if the discontinuous transition is the main interest.

\section{Acknowledgment}

S.-C.P. would like to thank the hospitality of Korea Institute for Advanced Study where this project was planned during his visit in 2007. This work has been supported by DFG within SFB 680 Molecular Basis of Evolutionary Innovations.
[1] T. Ohtsuki and T. Keyes, Phys. Rev. A 35, 2697 (1987).

[2] T. Ohtsuki and T. Keyes, Phys. Rev. A 36, 4434 (1987).

[3] H.-K. Janssen, J. Phys.: Condens. Matter 17, S1973 (2005).

[4] S. Lübeck, J. Stat. Phys. 123, 193 (2006).

[5] P. Grassberger, J. Stat. Mech.:Theory Exp. (2006), P01004.

[6] H. Hinrichsen, e-print arXiv:cond-mat/0006212

[7] R. Dickman and T. Tomé, Phys. Rev. A 44, 4833 (1991).

[8] H. Hinrichsen, Adv. Phys. 49, 815 (2000).

[9] G. Ódor, Rev. Mod. Phys. 76, 663 (2004).

[10] S. Lübeck, Int. J. Mod. Phys. B 18, 3977 (2004).

[11] C. E. Fiore and M. J. de Oliveira, Phys. Rev. E 70, 046131 (2004).

[12] G. O. Cardozo and J. F. Fontanari, Eur. Phys. J. B 51, 555 (2006).

[13] D. S. Maia and R. Dickman, J. Phys.: Condens. Matter
19, 065143 (2007).

[14] A. A. Ferreira and J. F. Fontanari, J. Phys. A: Math. Gen. 42, 085004 (2009).

[15] I. Jensen, J. Phys. A: Math. Gen. 29, 7013 (1996).

[16] M. Henkel and H. Hinrichsen, J. Phys. A: Math. Gen. 37, R117 (2004).

[17] S.-C. Park and H. Park, Eur. Phys. J. B 64, 415 (2008).

[18] S.-C. Park and H. Park, Phys. Rev. E 73, 025105(R) (2006).

[19] S.-C. Park and H. Park, Phys. Rev. E 76, 051123 (2007).

[20] I. D. Lawrie and S. Sarbach, in Phase Transitions and Critical Phenomena, edited by C. Domb and J. L. Lebowitz (Academic Press, London, 1984), vol. 9.

[21] H. J. Hilhorst and F. van Wijland, Phys. Rev. E 65, 035103(R) (2002).

[22] M. J. de Oliveira, Phys. Rev. E 67, 027104 (2003). 